\title{
THYROID DYSFUNCTION IN CHILDREN, ITS DIAGNOSIS, AND TREATMENT
}

\author{
By Zina E. MoncriefF, M.A., M.R.C.P.
}

Disease of the thyroid gland in children differs sufficiently from thyroid dysfunction in adults to warrant some special study. It is not common, and yet, with the exception of adenomatous goitre with hyperthyroidism, all the major diseases of the thyroid gland, which affect adults, have been seen in children. They may be divided for convenience into two groups, non-endocrine, and endocrine disorders.

\section{Non-Endocrine Conditions}

Congestion. A temporary hyperaemia of the thyroid gland may occur at birth, puberty, menstruation, or during the course of an acute illness. The gland becomes enlarged, firm and may be tender. The swelling subsides without any treatment, though cold compresses may hasten its disappearance.

Acute thyroiditis. This is uncommon in children. It may result from the toxaemia of an acute infection, or may be due to actual bacterial invasion of the gland. It may occur in a thyroid, which was previously normal, or be superimposed on a goitrous gland (strumitis). The thyroid presents the features of an acute inflammation, and the general signs of any acute infection are present. The gland becomes acutely swollen, and tender, and swallowing is usually painful. The swelling may be large enough to produce symptoms of pressure, and then dyspnoea may be severe, and distension of the neck vessels, with cyanosis of the face and neck may be present:

Treatment. It is that of any acute inflammatory process with local applications of heat to the gland, and general measures to combat the infection. The patient should be kept at rest in bed, and fluids taken copiously. If dyspnoea is present, a tracheotomy set should be kept ready at the bedside, for a tracheotomy may become necessary. Sulphamezathine or penicillin should be given in adequate dosage.

Acute thyroiditis generally subsides within the course of a few days, but some cases may go on to suppuration (suppurative thyroiditis). The treatment for this is surgical.

Chronic thyroiditis. This condition is vety rare in children. The gland is enlarged and firm and may be nodular There are no signs of endocrine disturbance, though in some recorded cases a slightly lowered basal metabolic rate has been noted. The diagnosis may be ascertained in some cases only after studying sections of the gland after subtotal thyroidectomy.

Carcinoma. This is a rare occurrence in a normal thyroid gland. It is met with more frequently as a secondary change in an adenomatous goitre. Cases occur at any age, and twice as commonly in females.

The danger signals are :

I. A change in consistency from a soft to a hard, irregular, nodulated swelling, generally in one portion of the gland.

2. An enlargement of cervical lymph nodes.

3. The development of hoarseness, dysphagia, or dyspnoea not due to obvious pressure from the size of the gland.

Treatment. This is surgical with subsequent irradiation therapy.

\section{Endocrine Disorders}

Simple or endemic goitre (colloid, parenchymatous, non-toxic). The simple hyperplasia 
which results from a low intake of iodine in the diet is known as simple goitre. In the districts, chiefly mountainous, where this disease is endemic, there is an increased rate of deaf-mutism, cretinism and idiocy.

The commonest age incidence is just before or at the time of puberty, though cases have occurred in which a goitre has asphyxiated the foetus in utero, and others in which the thyroid swelling produced a difficult delivery of the infant (congenital goitre). Girls are more affected than boys.

The thyroid presents a smooth, soft, symmetrical enlargement. The swelling varies in size from one which is scarcely appreciable to an enlargement which may cause marked pressure symptoms. The latter is more frequent in congenital goitre, and the critical period is during the first $4^{8}$ hours of birth. The swelling usually, though not always, diminishes in size after the first week of life.

Some of these cases develop signs of hypothyroidism, and in others carcinomatous changes occur.

\section{Treatment}

Prophylactic. Endemic goitre ranks as one of the most striking examples of a preventable disease. By the addition of iodine to the diet in goitrous areas the disease can be prevented. To this measure should be added the improvement of bad hygiene and poor living conditions, for McCarrison showed that in some areas the drinking water is bacterially contaminated, and that boiling and filtering of the water may prevent the disease.

The thyroid gland has the power to take up iodine from any of the known compounds of the substance, irrespective of the method of administration. The method of choice, therefore, should provide a compound which is palatable, cheap, harmless and easy to administer. The most satisfactory method of administration of iodine is in iodized table salt. It is used in place of ordinary table salt, and the recommended proportion is I part of sodium iodide to ro,000 parts of salt. Any child consuming an average amount of this table salt per day will be protected against the disease.

Other methods of administration are available. Sodium iodide $0.13 \mathrm{gm}$. (2 gr.) may be given daily for two consecutive weeks every spring and autumn, or Lugol's solution one drop weekly throughout the year. Chocolatecoated tablets of iodostarine, a combination of organic iodine and a vegetable fatty acid compound are available, the dose being $\frac{1}{2}$ to one 5 mgm .tablet once a week.

It is important that the foetus should be protected against this disease, and it is recom: mended that an extra intake of iodine be ingested by the pregnant woman in endemic areas.

Curative. Once the colloid stage has developed, iodine is of limited value. It is worthy of a trial, however, for, in some cases, a diminution in the size of the gland occurs. In Great Britain Lugol's solution 9-I5 drops are given daily, but in some parts of the world, particularly in Switzerland, and in certain parts of America, much smaller doses of iodine are given. Workers in these parts claim that there is a real danger of provoking symptoms of hyperthyroidism, if excessive quantities of iodine are administered (IodineBasedow). Other American workers, in areas of high goitre endemicity, do not record this complication.

When symptoms of hypothyroidism are present adequate dessicated thyroid substance should be given. The size of the gland will not be reduced by this treatment.

Surgery is indicated if the swelling is objected to on aesthetic grounds, or if pressure symptoms appear; also if a nodular swelling develops in the goitre (this is potentially malignant), or if there arises the suspicion of carcinomatous change.

Irradiation therapy is contraindicated, for it may result in hypothyroidism.

\section{Adenoma of the Thyroid}

Adenomatous goitre occurs most commonly in goitrous areas. It has been recorded in all age groups, with the greatest number occurring in older children. As with other varieties of goitre, females are more affected than males. The adenomata vary in size and number, and may cause symptoms of pressure. No case of hyperthyroidism in an adenomatous goitr: has been recorded in childhood.

Treatment. Some of these cases have shown a diminution in the size of the goitre after the (Continued on page 42.) 


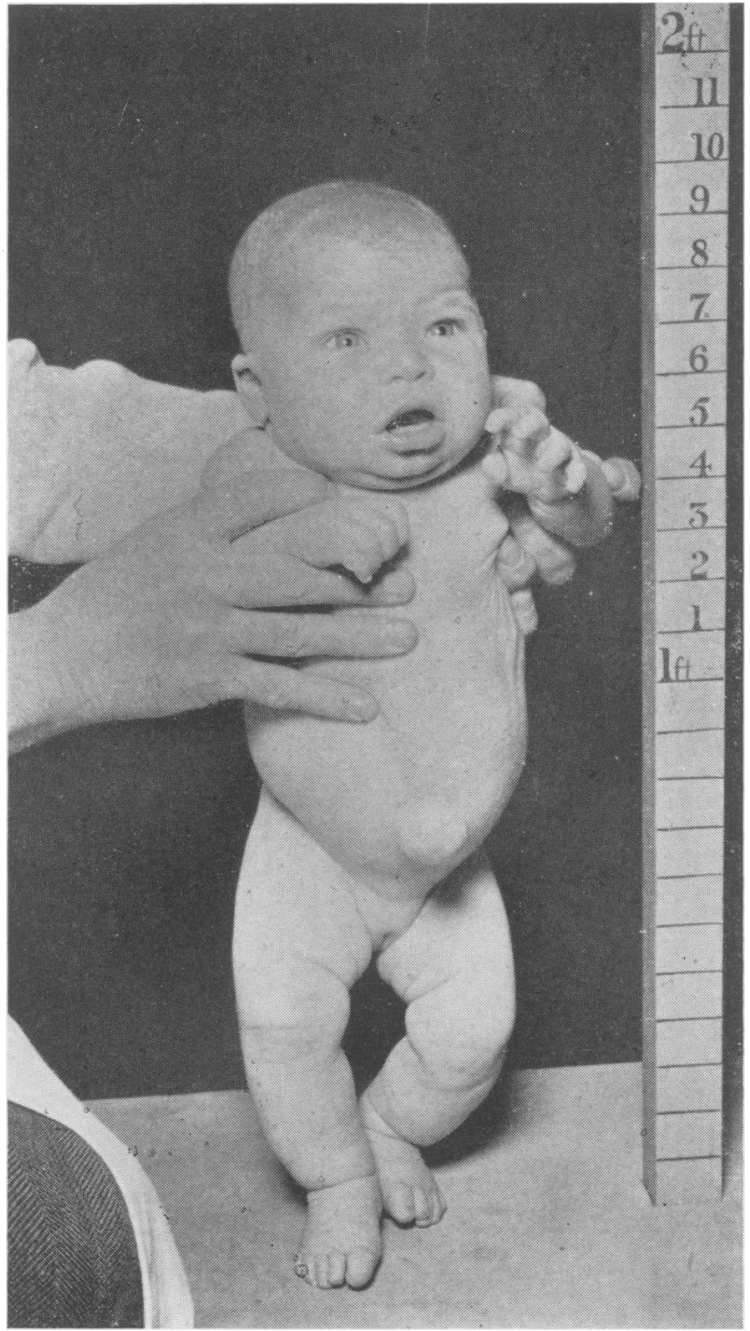

FiG. 1.-Cretin aged six months. 


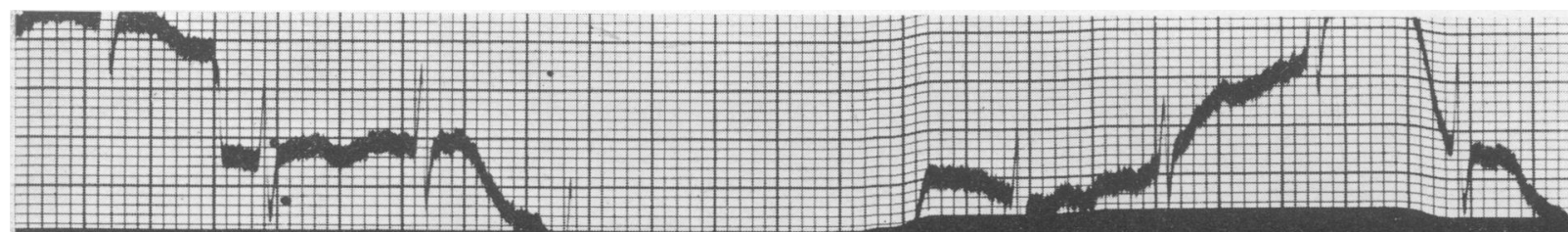

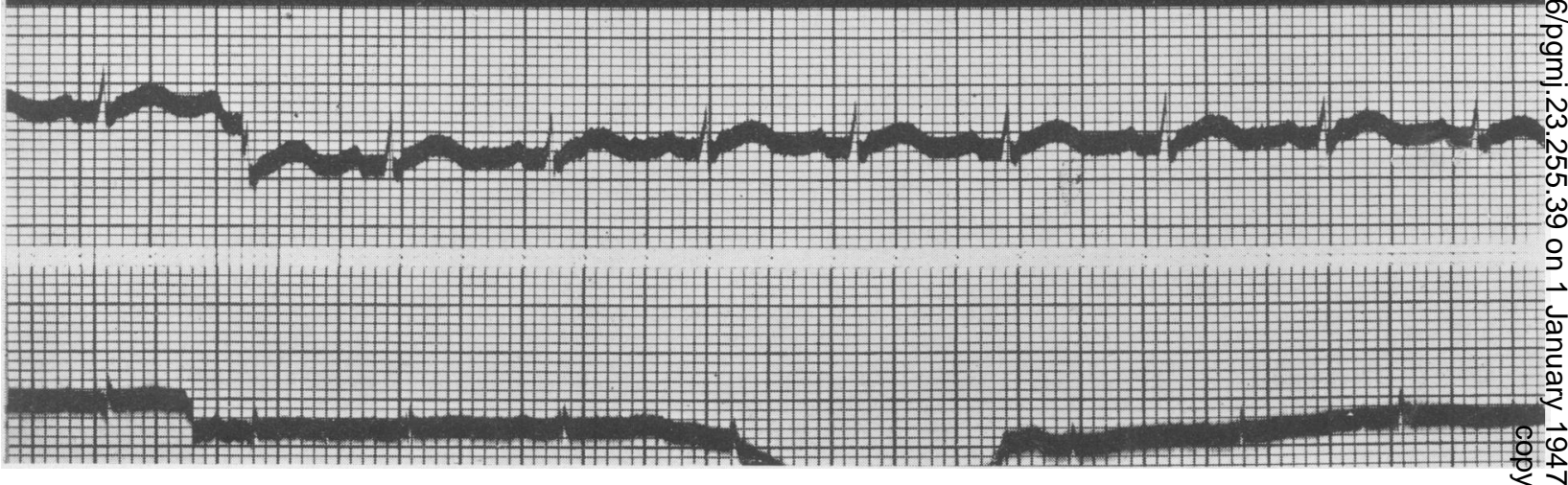

FIG. 2.-Electrocardiogram of cretin in Fig. I, showing low voltage.

(Continued from page 40.)

administration of iodine, or of dessicated thyroid, or of both given at the same time. Others have shown no improvement. If a definite nodular swelling persists, then surgical removal should be advised, for adenomata of the thyroid show a high rate of malignancy. Enucleation of the adenoma, lobectomy, or subtotal thyroidectomy may be carried out.

\section{Cretinism}

Cretinism occurs in Great Britain in sporadic form, and in goitrous areas it appears endemically. Endemic cretins may have the signs of the disease at birth. In cases of sporadic cretinism, however, the symptoms rarely appear before the twelfth week of life.
The child is afforded the protection of the mother's secretion in utero, and it takes four to eight weeks after birth for the store of thyroxin, obtained from the mother, to be depleted. Instances have been recorded, however, of women with goitre giving birth to infants recognizable as cretins from birth.

The earliest sign of the disease is a failure to grow. It'soon becomes apparent that there is a failure in mental development as well. Constipation, however, may be the first symptom which comes to the mother's notice, and which causes her to seek medical advice. The hoarse, rough cry of a cretinous infant is very characteristic, and may lead one to look elsewhere for the other symptoms or signs of

(Continued on page 45.) 


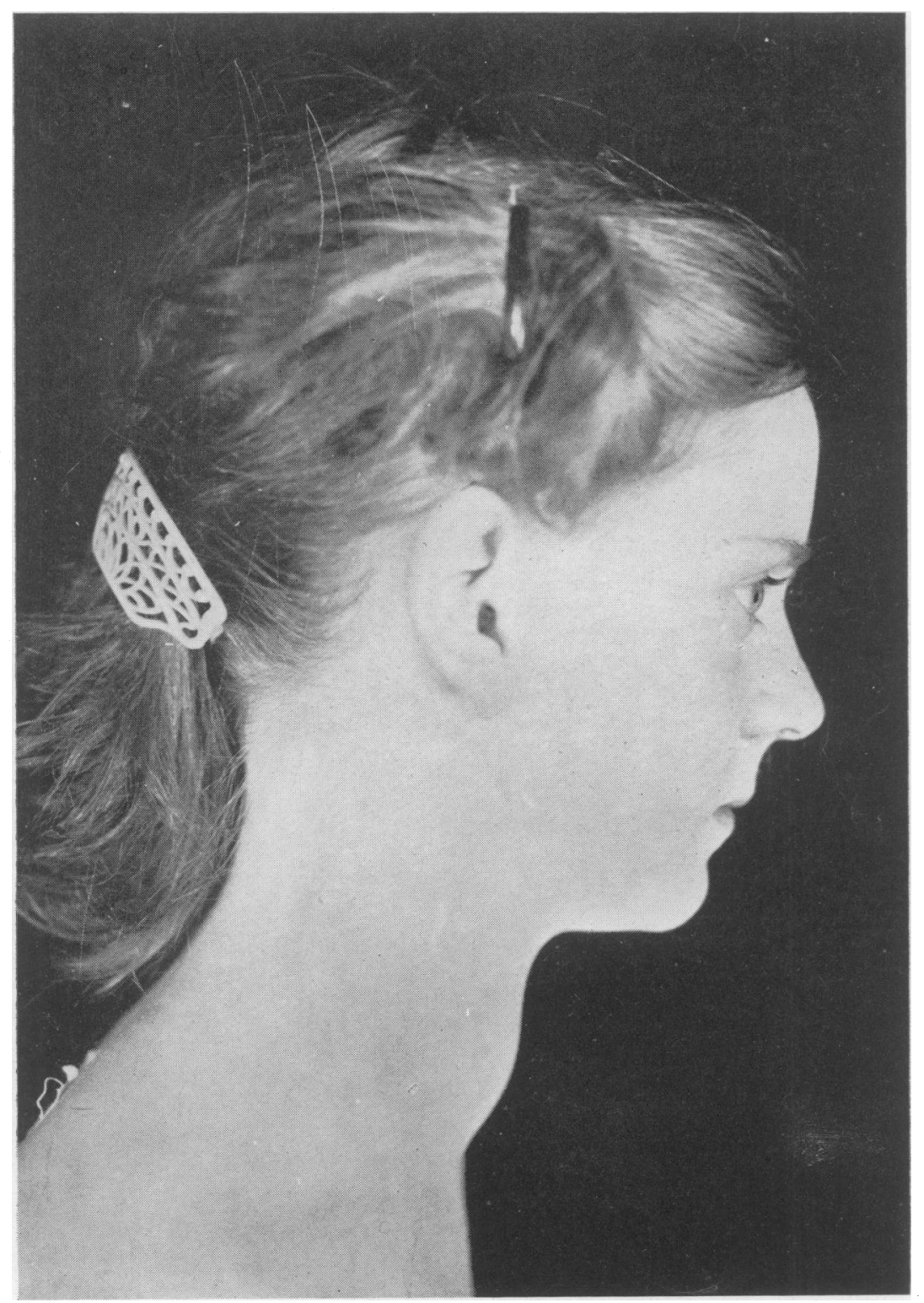

Fig. 3.-Toxic goitre in a girl of to years of age. 


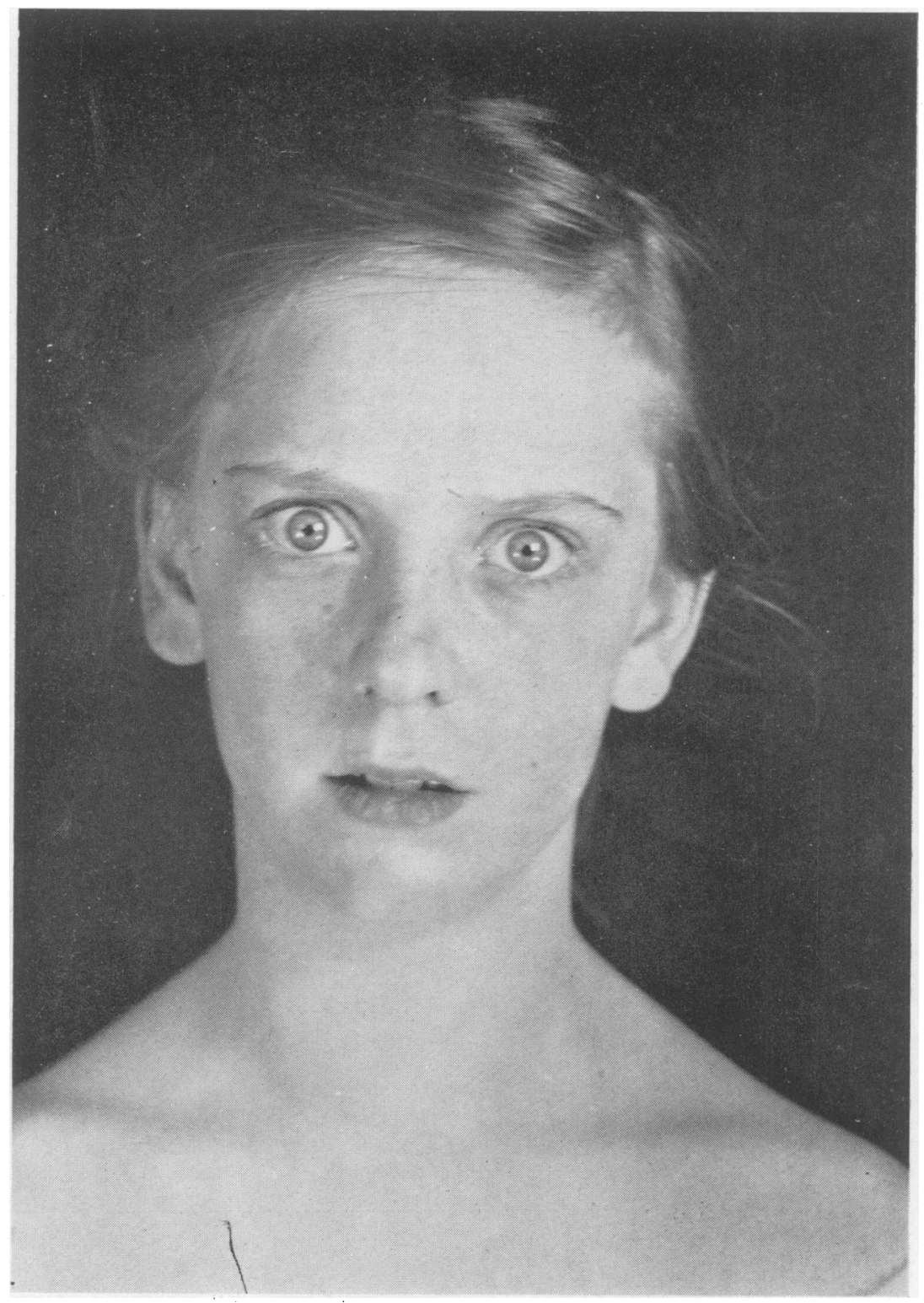

Fig. 4.-Same child, showing the exophthalmos.

I am indebted to Dr. Chas. Donald and to the Hospital for Sick Children, Great Ormond Street, for permission to publish these photographs. 
(Continued from page 42.)

the disease. Even in very young cretins the thickening of subcutaneous tissues is usually already present when the child is first seen. It produces the typical facies which makes the diagnosis unmistakeable in the fully developed case. The face is puffy, and the eyelids thickened, and the tongue is enlarged and protrudes from the half open mouth. There is often broadening at the root of the nose with narrow palpebral fissures. In addition to this, the hair may be coarse and dry, and the skin is thickened and may show eczematous patches. The abdomen is usually protuberant and an umbilical hernia is present. The extremities may be blue and cold, as the result of the poor circulation, and there may be marked hypotonicity of muscles. In older cretins fatty tumours are frequently present. They are about the size of a hen's egg, and are placed symmetrically in certain positions. Above the clavicles, behind the sternomastoid muscles, in the axillae and between the scapulae are the common sites. There may be evidence of an anaemia.

\section{Later Manifestations}

In older cretins there may be clinical evidence of a delay in ossification. The anterior fontanelle which normally closes by the end of the eighteenth month may remain patent into adult life. Infantile proportions of the skeleton may persist. (That is, the mid point of the total height is about the level of the umbilicus). Dwarfism' is always marked, and an untreated cretin rarely grows higher than 3 to $4 \mathrm{ft}$., and is often much shorter. The teeth erupt late in the case of both dentitions, and are of poor quality. Secondary sex characteristics may never appear.

The energy exchange in cretinism is small and these children are very susceptible to cold. The temperature tends to be subnormal and the pulse slow. The blood pressure is low.

\section{Other aids to diagnosis}

It is difficult to record an accurate estimation of the basal metabolic rate in cretins. Other methods are available, however, for confirming the diagnosis.

r. Radiograms of the carpal bones will show delayed ossification. (Normally one centre of ossification appears in the wrist per year for the first seven years of life, excepting in the fourth year.)

2. The blood cholesterol is raised $(N=\stackrel{\varrho}{c}$ I50-230 mgms. per cent.) It is inversely $\Rightarrow$ proportional to the B.M.R. Its return to normal forms a good criterion of the response to thyroid therapy.

3. The total blood lipoids are increased.

4. There is an absence or diminution of creatinin in the urine.

5. There is a therapeutic response to the administration of thyroid substance and, in difficult cases, this may be the most helpful confirmatory measure. It produces a brisk reticulocytosis after ten days administration, and this fact may be used as an early diagnostic factor. The improvement in the general condition may be seen after one week's therapy.

\section{Differential diagnosis}

I. Mongolism is often discussed as presenting a possible difficulty in the differential diagnosis from cretinism. The two conditions are quite dissimilar. Once a mongol child with its slanting eyes and brachycephalic skul has been recognized, there can be no difficult about the diagnosis in future cases.

2. Gargoylism at first sight is usually mistaken for cretinism. In this condition, however, as well as mental deficiency and dwarfism, there are various eye defects, and enlargement of the liver and spleen and an osteodystrophy. There is no evidence of a delay in ossification. Radiograms of the lumbar spine and the heads of long bones show characteristic changes.

3. The individual features of cretinism may be simulated as in macroglossia, and ichthyosis, but other features of the disease are not present.

Treatment. When thyroid substitution therapy is begun and maintained from the early months of the disease, the results of treatment may be satisfactory. The prospects of relief of symptoms, however, steadily diminish with the increase in age of the untreated patient, and this is most marked in the alleviation of mental retardation. Treatment begun after the child has reached the age of one year will produce a marked physical improvement, but the mental condition will never be normal. If left untreated, cretins become dwarfs 2 to $3 \mathrm{ft}$. high with a gross amount of , 
mental defect. Treatment begun in older children may rouse a placid cretin into a state of peevish irritability, and, in others, symptoms of hyperthyroidism may be induced.

The correct dose of thyroid extract is that amount which will produce normal growth and development, without any symptoms of hyperthyroidism. The substance must be administered only under medical supervision. Parents must be instructed that the treatment must continue throughout the child's lifetime, for an interval of some months without treatment may produce mental impairment, which is never correctable.

Thyroid substance begins to act within three to seven days of ingestion and, when continuous daily doses are taken, the maximal effect may be delayed until the end of the third week. Signs of toxic reaction to the drug in cretins are not unusual, and this may appear after the exhibition of a single dose or during the course of thyroid therapy, which has continued for several years. Bearing these facts in mind, a useful scheme of dosage is the following :

Extract thyroid sicc. gr. I/Io t.d.s. for one week.

Extract thyroid sicc. gr. I/8 t.d.s. for a further week.

Extract thyroid sicc. gr. I/4 t.d.s. for one more week, and so on.

The correct maintenance dose of thyroid extract is obtained by producing signs of hyperthyroidism, it is then reduced and it is that amount at which these signs disappear. The signs of hyperthyroidism may be diarrhoea a rise in the body temperature an increase in the pulse rate, sweating, irritability, disturbed sleep, or a marked loss of weight. An initial loss of weight is common, but this should never be allowed to become drastic. If any of these signs become severe, the drug should be withdrawn completely until they disappear, and a start then made again with a reduced amount. If, however, any of these signs appear and are not sufficiently severe to affect the child's general condition, the drug should be persevered with.

Care should be taken to ensure that the thyroid substance which is being administered is potent.

As growth is likely to be rapid, it is important that big doses of vitamins, particularly of vitamin $D$, should be given with regularity.

If there is any anaemia present, it is likely to be of a simple nutritional type. Iron may be given as iron and ammonium citrate, or as ferrous sulphate, in a mixture starting with small doses and working up to relatively large amounts by daily increases.

\section{Hypothyroidism-Infantile Myxoedema}

Thyroid deficiency may become apparent at some time after birth, generally not before the second year. The history is frequently that the child has been developing normally, and then, often after some acute illness, a delay in growth and signs of mental retardation appear. He shows an inability to learn and may lose the ability to do the things he had formerly acquired. He becomes dull, lethargic, and unclean in his habits. He often complains readily of cold.

The signs of the disease are essentially those of cretinism. In severe cases it is hardly possible to err in the diagnosis, but when the disease is mild, or of short duration, the diagnosis may be made only by the therapeutic response to the giving of thyroid.

The rules for treatment are similar to those already indicated for cretinism. Prompt treatment produces a striking relief of symptoms, and physical growth and intellectual attainment may become normal. Delayed, or inadequate therapy will bring about mental retardation, which can never be corrected.

As in cretinism, a generous allowance of vitamins must be provided, and iron should be given to correct any anaemia.

\section{Hyperthyroidism (Toxic, exophthalmic Goitre)}

This is an uncommon disease in children. It has been recorded in the infant of a mother suffering from toxic goitre, but it is unusual before the age of eight years. The commonest age incidence is before puberty. It is commoner in girls in the proportion of $5: \mathrm{r}$.

The clinical picture is essentially the same as that seen in adults, but the disease appears more suddenly and disappears with much 
greater rapidity. The late results of untreated, or improperly treated cases, are seen far less frequently than in adults. Permanent damage to the heart has not been recorded. Crises are less frequent and less severe. Nervousness is the most frequent complaint, followed by tachycardia, goitre, and exophthalmos in that order. Nervousness associated with an enlargement of the thyroid gland in children is very suggestive of this disease. The child usually becomes irritable, emotionally unstable, and unmanageable. She is often constantly in motion with chorea-like movements and can be kept quiet only with difficulty. Vivacity is usually a marked feature. Augmentation of the height is common, yet there is usually a loss of weight, associated with an excellent appetite. Some of these patients gain weight. Diarrhoea is common.

On examination, four-fifths of the cases show exophthalmos, and Stellwag's and von Graefe's signs are usually present. The skin may be warm and moist. The fine tremor of the hands seen in adults is usually missing. It is a coarse tremor and may be accompanied by coarse involuntary movements. The goitre is a diffuse enlargement of one or both lobes, and a bruit can be heard on auscultation. Almost every case of this disease, though not all, shows an enlargement of the gland. It may be sufficiently enlarged to come to the notice of the parents, or may be discovered only after close scrutiny. Tachycardia is likewise almost always present, and the child may complain of the violent action of the heart. There may be some evidence of cardiac dilatation. The systolic blood pressure is raised, with a resultant high pulse pressure. A disturbance in carbohydrate metabolism may be apparent by occasional glycosuria.

\section{Investigations}

I. The basal metabolic rate is raised, and it is often higher than the clinical picture would warrant one to expect. It can be estimated without difficulty as a rule, and it forms an excellent measure of severity and progress.

2. Radiograms may show an advanced bone age.

3. The blood cholesterol is diminished.

4. Some workers have shown that there is a lack of sensitivity to the action of quinine $\frac{2}{z}$ They claim that quinine sulphate, 5 gr. give three times a day for some days, will not produce the signs of cinchonism.

\section{Medical Treatment}

Treatment. In mild cases, and in those of short duration medical treatment is justified? If a prompt subsidence of symptoms and signs occurs then it may be continued. Attentiom should be directed towards securing mentat and physical rest. It may be sufficient to keew the child at home with extra rest and with a adequate intake of a sedative. In less mile. examples of hyperthyroidism, it may be necessary to keep the child in bed completeliv at rest. It may be wise to remove the child from its home, away from family discussions quarrels and extraneous noists. If the disease is of any severity, contact with other children must be avoided. Anything calculated to irritate or excit $\epsilon$ the patient must be eliminated She must be protected from intercurrent in fection. Attention must b directed towablso the eradication of any septic focus, but aisy operative procedures must be permitted ow during a quiescent period of the hypet? thyroidism.

Sedatives. Phenobarbitone is still one of the most effective drugs for producing a quize state in this condition. Children tolerate $i \overrightarrow{\vec{b}}$ well and there is no cumulative action. The dose varies from $\frac{1}{8} \mathrm{gr}$. in small children tof $\frac{1}{2}$ gr. at the age of twelve years given three times a day. Older children may be given large doses with impunity. It may be giveng with a potassium bromide and chloral hydrate mixture. Other drugs which may be tried are nembutal and amytal. Whatever drug is chosen, enough of it must be given to keep the patient quiet, without producing any toxie manifestations.

Diet. It should provide a high caloric intake $\stackrel{N}{N}^{\circ}$ and, in most cases, be unrestricted in variety Protein is necessary to make good the tissuew waste inevitable with a high metabolic rate, but should not be given in excess, because of? its specific dynamic action.

Iodine. Much difference of opinion existsover the advisability of administering iodine too patients suffering from hyperthyroidism. 
Many mild cases will be cured by employing the treatment which has been outlined. It must be remembered that others achieve a spontaneous cure at puberty. When the symptoms of the disease are not checked by a short course of rest and sedatives. then it is justifiable to administer iodine. In children its maximal effect may not become apparent for two to three weeks. As little as $2 \mathrm{~m}$. Lugol's solution three times a day will produce the optimum effect, and larger doses of the mixture do not enhance its beneficial action. It may be given in courses of from one to three weeks, the drug being withdrawn between courses when it becomes apparent that the optimum effect has been obtained. The response to iodine is never so dramatic as it is after the first course. In some cases sufficient improvement in the nervous and toxic symptoms and in the B.M.R. is maintained after each course to warrant the continued administration of iodine. In others it becomes evident that the disease has become refractory to further dosage and the drug should be withdrawn.

No one will challenge the undoubted value of iodine in pre-operative and post-operative treatment. Lugol's solution, $2 \mathrm{~m}$. t.d.s., should be given for the three weeks immediately pre-ceding operation.

Thiouracil. This drug may be used in the treatment of thyrotoxicosis per se in mild cases; or as a pre-operative measure. The preparation of patients for surgery can usually be accomplished with this drug in eight weeks or less. Its employment in children has not yet been fully evaluated. Three results, however may be looked for.

I. The drug may produce no effect. Doses employed have varied, but average about 0.1 or $0.2 \mathrm{gm}$. per day. If there has been no previous iodine therapy, medication should be continued for at least 30 days or, if iodine has been administered, for 60 to 100 days, before concluding that no response to thiouracil will occur.

2. The disease may be controlled and the basal metabolic rate may be reduced or even fall to within normal limits. The amount of thiouracil is then reduced by at least half and this may be continued as a maintenance dose.
Information is still lacking as to the permanence of improvement in the disease.

3. Toxic manifestations may appear. The danger period is within the first 12 weeks of treatment, and from four to eight weeks most complications have been recorded. In patients who have received previous treatment with thiouracil these may appear as early as the second week. The incidence of toxic reactions up to nine months make continued supervision essential during administration.

The reactions to be looked for are granulocytopenia, leucopenia, fever, skin lesions, lymph node enlargement, jaundice, purpura and anaemia. Parents must be instructed to report at once the occurrence of sore throat, fever, cold in the head or malaise, and any other untoward symptom which may occur. Toxic reactions appearing early call for a withdrawal of the drug. Blood changes are always of serious significance and they indicate an instant cessation of the treatment. It is important to note the fact that toxic manifestations may appear during a second course of thiouracil, although the first course was given without incident.

\section{Surgical Treatment}

In any but the mild cases of this disease surgical intervention is advisable. Children tend to react severely to surgical operation but the pre-operative treatment with iodine and, more recently, thiouracil, has materially affected the mortality rate. The operation is subtotal thyroidectomy. At the Mayo Clinic I 57 children suffering from hyperthy;oidism have been treated. Of these 136 were treated surgically, and surgical intervention was recommended, but did not take place, in some of the remaining 21 cases. Before the introduction of iodine the surgical mortality rate at the Clinic was estimated at 9 per cent., and, since iodine has been used pre-, and postoperatively, this figure has been reduced to 2.5 per cent.

These patients should be observed at frequent intervals for one to two years after operation. Some of them develop signs of hypothyroidism; others show a recurrence of thyrotoxicosis. A watch should be kept, therefore, and if symptoms appear, the appropriate treatment should be cairied out. 